\title{
A Hierarchical Geometric Framework to Design Locomotive Gaits for Highly Articulated Robots
}

\author{
Baxi Chong*, Yasemin Ozkan Aydin*, Guillaume Sartoretti ${ }^{\dagger}$, Jennifer M Rieser*, Chaohui Gong ${ }^{\dagger}$, Haosen Xing ${ }^{\dagger}$, \\ Howie Choset ${ }^{\dagger}$ and Daniel I Goldman * \\ ${ }^{*}$ Georgia Institute of Technology, ${ }^{\dagger}$ Carnegie Mellon Institution
}

\begin{abstract}
Motion planning for mobile robots with many degrees-of-freedom (DoF) is challenging due to their highdimensional configuration spaces. To manage this curse of dimensionality, this paper proposes a new hierarchical framework that decomposes the system into sub-systems (based on shared capabilities of DoFs), for which we can design and coordinate motions. Instead of constructing a high-dimensional configuration space, we establish a hierarchy of two-dimensional spaces on which we can visually design gaits using geometric mechanics tools. We then coordinate motions among the two-dimensional spaces in a pairwise fashion to obtain desired robot locomotion. Further geometric analysis of the two-dimensional spaces allows us to visualize the contribution of each sub-system to the locomotion, as well as the contribution of the coordination among the sub-systems. We demonstrate our approach by designing gaits for quadrupedal robots with different morphologies, and experimentally validate our findings on a robot with a long actuated back and intermediate-sized legs.
\end{abstract}

\section{INTRODUCTION}

High degree-of-freedom (HDoF) mobile robots offer advantages over conventional wheeled vehicles in difficult-tonegotiate terrains $[3,17,21,25]$. However, the benefit of many degrees of freedom also poses a challenge: how to coordinate all of the DoFs to produce purposeful motion. One approach to address this so-called curse of dimensionality is to couple groups of the DoFs into one or two DOFs thereby producing a lower dimensional configuration space $[5,10,15,16,24,2,6]$. We term these lower dimensional systems as sub-systems. Subsystems can be formed based on observations of biological systems, empirical experiments, or intuition. The approach described in this paper first plans for motions within every sub-system, and then for coordination among the sub-systems. Specifically, we propose a hierarchical framework to decompose the motion planning problem in the full configuration space of a robot, into i) a series of simpler motion planning problems in the low-dimensional spaces associated with the different sub-systems, and ii) the coordination of these subsystems.

Our framework relies on a motion planning approach, namely geometric mechanics (GM), which offers powerful tools to design, visualize, and analyze a wide range of locomotor behaviors $[4,7,14]$. We rely on GM tools to visually design the motion of each subsystem with respect to our locomotive goals (here, to maximize stride displacement), and to design the coordination of pairs of sub-systems. By relying on a coordinate-descent-like approach, we then optimize locomotion by iterating over every pair of sub-systems until

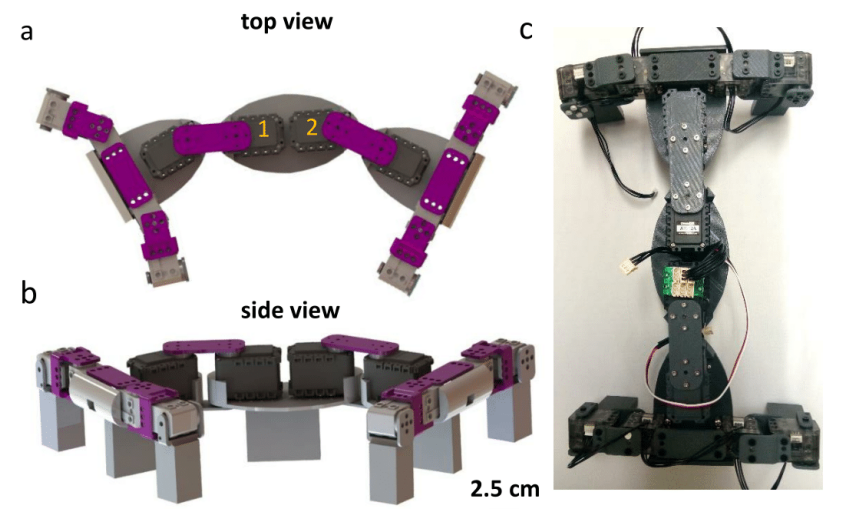

Fig. 1. Three link swimmer with four cubic legs CAD diagram of the robot (a) top and (b) side view. (c) Physical model. The upper back and lower back are actuated by two AX12 servos (labeled as 1 and 2) and the legs are actuated by XL320 servos. The legs have rectangular shape $(24 \times 24 \times 45 \mathrm{~mm})$ and the body is always in contact with ground via blocks $(55 \times 12 \times 45 \mathrm{~mm})$ attached to the body segments.

convergence is attained. Once this process has converged, we further show how GM tools allow us to visualize the contribution of each sub-system to locomotion, as well as that of the coordination of every pair of sub-systems. In this way, the proposed hierarchical framework provides a motion planning tool to visually and intuitively design gaits for $\mathrm{HDoF}$ systems.

We apply our framework to study the motion of a quadrupedal robot with a long articulated body and intermediate-sized legs (Fig. 1), a morphology incorporating features from both lizards and snakes. Here, we show how our framework provides insight into the role of leg contacts and body undulation in quadruped locomotion. Previous work has shown that the undulation of an elongated body improves the stride length of animals and robots [11, 27, 8]. A more comprehensive review on quadrupedal gait design can be found in [27]. There are two common modes of body undulation: standing waves (lizard-like) [11, 27], and traveling waves propagated from head to tail (snake-like) [8]. In the first mode, the body curvature is maximized to increase the selfpropulsion generated by leg-ground interaction. In the second mode, the body undulation can generate self-propulsion even without legs. Interestingly, we find that the optimized wholebody motion (i.e., motion in the full configuration space) for our robot is a hybrid combination of the lizard- and snake-like modes, such that the self-propulsion from leg-ground contact and that from body-ground contact are properly coordinated. 

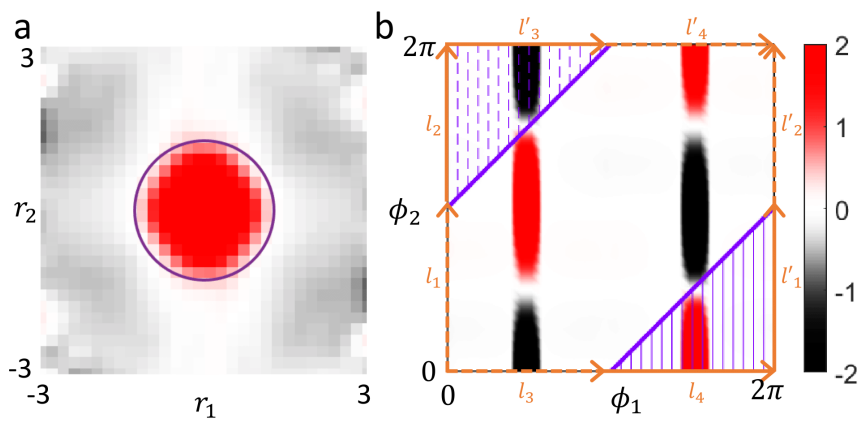

Fig. 2. Example height functions and gaits in different shape spaces (a) The height function in a Euclidean shape space corresponds to motions of an 8-link snake robot slithering in the forward direction on the surface of $6 \mathrm{~mm}$ plastic particles. The purple circle represents the optimal gait in the corresponding shape space. (b) The height function in a torus shape space corresponds to the forward motion of a robot with a long actuated body and intermediate-sized legs on the surface of $\sim 1 \mathrm{~mm}$ poppy seeds. The solid purple curve represents a sample gait in the corresponding shape space. Orange lines represent the assistive lines to form closed loops with the gait path in the unfolded torus shape space. The area in the solid purple shadow represents the area where the gait path and the assistive lines form a clockwise loop; the area in the dashed purple shadow represents the area where the gait path and the assistive lines form a counterclockwise loop. Red, white and black indicate positive, zero and negative values in height function respectively.

We validate our theoretical predictions through a series of experiments where a 10-DoF robot locomotes on granular material.

The paper is structured as follows: Section II introduces geometric mechanics and presents our framework to design whole-body motion; Section III applies our methods to study the motion design of a robot with a long articulated body and four legs; Section IV discusses the significance and concludes our work.

\section{METHOD}

\section{A. Review of geometric mechanics}

In this section, we provide a concise overview of the geometric tools needed for this paper. For a more detailed and comprehensive review, we refer readers to [4, 7, 14]. The gait design tools of geometric mechanics separate the configuration space of a system into two spaces: the position space and the shape space. The position space denotes the location of a system relative to a world frame, while the shape space denotes the internal configuration (internal shape) of the system. Geometric mechanics seeks to establish a functional relationship between velocities in these spaces; this functional relationship is often called a connection, and it shares many properties with the Jacobian of a robotic manipulator.

1) Kinematic reconstruction equation: Principally, in kinematic systems where there is no drift in the system, the equations of motion [14] reduce to

$$
\boldsymbol{\xi}=\boldsymbol{A}(\boldsymbol{r}) \dot{\boldsymbol{r}},
$$

where $\boldsymbol{\xi}=\left[\begin{array}{lll}\xi_{x} & \xi_{y} & \xi_{\theta}\end{array}\right]^{T} \in g$ denotes the body velocity in forward, lateral, and rotational direction; $\boldsymbol{r}$ denotes the internal shape variables (joint angles); $\boldsymbol{A}(\boldsymbol{r})$ is the local connection matrix that relates shape velocity $\dot{\boldsymbol{r}}$ to body velocity $\boldsymbol{\xi}$. Eq.(1) is also called the kinematic reconstruction equation and maps the changes in internal shape variables (joint angles) to changes in group variables (position and orientation) of the robot.

The interaction between a robot and an environment is often difficult to model, which is typically the case when the system locomotes on a granular medium. In such a case, calculating the local connection matrix $\boldsymbol{A}$ can be quite challenging. Instead, we numerically derive $\boldsymbol{A}$ using resistive force theory (RFT) $[13,22,26]$ to model the contact. Prior work [8] has shown that numerically derived local connections using granular RFT can effectively predict movements in granular media. In this paper, we model the robot-ground contact by poppy seed RFT equations [27], from which we numerically derive the local connections.

2) Connection vector fields and height functions: Each row of local connection matrix $\boldsymbol{A}$ corresponds to a component direction of the body velocity and therefore gives rise to a connection vector field. The body velocities in the forward, lateral and rotational directions are respectively computed as the dot product of connection vector fields and the shape velocity $\dot{\boldsymbol{r}}$. A shape velocity $\dot{\boldsymbol{r}}$ along the direction of the vector field yields the largest possible body velocity, while a shape velocity $\dot{\boldsymbol{r}}$ orthogonal to the field produces zero body velocity.

A gait can be represented as a closed curve in the corresponding shape space. The displacement resulting from a gait, $\partial \chi$, can be approximated by

$$
\left(\begin{array}{c}
\Delta x \\
\Delta y \\
\Delta \theta
\end{array}\right)=\int_{\partial \chi} \boldsymbol{A}(\boldsymbol{r}) \boldsymbol{d r}
$$

According to Stokes' Theorem, the line integral along a closed curve $\partial \chi$ is equal to the surface integral of the curl of $\boldsymbol{A}(\boldsymbol{r})$ over the area enclosed by $\partial \chi$ :

$$
\int_{\partial \chi} \boldsymbol{A}(\boldsymbol{r}) \boldsymbol{d} \boldsymbol{r}=\iint_{\chi} \nabla \times \boldsymbol{A}(\boldsymbol{r}) \boldsymbol{d} r_{1} \boldsymbol{d} r_{2},
$$

where $\chi$ denotes the area enclosed by $\partial \chi$. The curl of the connection vector field, $\nabla \times \boldsymbol{A}(\boldsymbol{r})$, is referred to as the height function (Fig. 2a). The three rows of the vector field $\boldsymbol{A}(\boldsymbol{r})$ can thus produce three height functions in the forward, lateral and rotational direction, respectively.

With the above derivation, we simplify the gait design problem to drawing a closed path in Euclidean shape space. The displacements can be approximated by the volume enclosed by the gait path. For example, in Fig. 2a, we show an example height function in Euclidean shape space corresponding to the motion in the forward direction of an 8-link snake robot slithering on the surface of $6 \mathrm{~mm}$ plastic particles. The circle (solid purple circle in Fig. 2a) enclosing the most volume represents the gait with the largest forward stride displacement.

Although the review of geometric mechanics sections are limited to two dimensions, there has been recent work on applying geometric mechanics to high-dimensional robot gait design [5, 20]. Ramasamy and Hatton [20] extended geometric mechanics to high dimensional shape space and proposed a 
method to visualized the kinematics. Gong et al. [5] approximated the kinematics of complex locomotors using only two shape variables with the proper shape basis functions.

3) Torus shape space: Often, a shape space can have some cyclic structure. With both shape variables being cyclic, the shape space has a torus shape, $\left(T^{2}\right)$ [12]. In Fig. 2b, we show an example height function in a torus shape space corresponding to the forward motion of a quadruped robot with the standing wave body undulation on the surface of $\sim 1$ $\mathrm{mm}$ poppy seeds. The shape variable, $\boldsymbol{\Phi}=\left[\phi_{1}, \phi_{2}\right]^{T} \in T^{2}$, has a cyclic structure, where one axis represents the phase of the leg movement, and the other axis represents the phase of the body undulation. While the gait path (solid purple curve Fig. 2b) is a closed curve in the torus shape space, there is no obvious area enclosed by the gait path.

To form an enclosed area, we introduce the notion of two assistive lines in the Euclidean parameterization of the torus shape space. The assistive lines are defined as: $\partial \chi_{1}: \phi_{1}=0$, (the solid orange line $l_{1}+l_{2}$ in Fig. 2b), and $\partial \chi_{2}: \phi_{2}=0$ (the solid orange line $l_{3}+l_{4}$ in Fig. 2b). Note that the dashed line $l_{i}^{\prime}$ is equivalent to the corresponding solid line $l_{i}$, according to the cyclic property of the torus shape space. In Fig. $2 b$, the gait path (the solid purple curve) together with two assistive lines $\left(l_{1}^{\prime}, l_{2}, l_{3}^{\prime}, l_{4}\right)$ encloses two areas in the torus shape space (solid purple shadow area and dashed purple shadow area). The net displacement can be approximated by the line integral along two assistive lines plus the surface integral of the enclosed area:

$$
\begin{aligned}
\int_{\partial \chi} A(r) d \Phi & =\int_{\partial \chi_{1}} A(\Phi) d \Phi+\int_{\partial \chi_{2}} A(\Phi) d \Phi \\
& +\iint_{\chi-\chi_{1}-\chi_{2}} \nabla \times A(\Phi) d \phi_{1} d \phi_{2},
\end{aligned}
$$

where $\chi-\chi_{1}-\chi_{2}$ denotes the area enclosed by the assitive lines and gait path. Note that in Fig. $2 b$, the gait path and the assistive lines form a clockwise loop in the lower right corner (solid purple shadow area) and a counterclockwise loop in the upper left corner (dashed purple shadow area). Taking the handedness into account, the surface integral (third term on the right hand side) in Eq.(4) should be computed as the surface integral of the enclosed area in the clockwise loop (solid purple shadow area in Fig. 2b) minus the surface integral of the enclosed area in the counterclockwise loop (dashed purple shadow area in Fig. 2b).

To interpret the physical meaning of each term (right hand side) in Eq.(4), we introduce the notation of two sets $P_{a}$ and $P_{b}$, where $P_{a}$ and $P_{b}$ denote the contribution from the horizontal and vertical coordinate axes in the torus shape space. In our example in Fig. 2b, $P_{a}$ represents the contribution from leg movement and $P_{b}$ represents the contribution from standing wave body undulation.

The physical meaning of the first term (right hand side) in Eq.(4) is the displacement solely resulting from motions in $\phi_{2}$, while keeping $\phi_{1}$ constant at the initial position, i.e., $P_{a}^{C} \cap P_{b}$, where ()$^{C}$ denotes the complement set. The physical meaning of the second term (right hand side) in Eq.(4) is the displacement solely resulting from motions in $\phi_{1}$, while keeping $\phi_{2}$ constant at the initial position, i.e., $P_{a} \cap P_{b}^{C}$. The first two terms (right hand side) in Eq.(4) are constant and independent from any gait path we choose. The physical meaning of the last term (right hand side) in Eq.(4) is the additional displacement resulting from the contribution from coordination of $\phi_{1}$ and $\phi_{2}$, i.e., $P_{a} \cap P_{b}$.

Finally, we refer readers to [7] for a detailed derivation and proof of motion planning in torus shape spaces.

\section{B. Whole-body motion}

1) Sub-system motion phase variable: In this section, we construct a high-dimensional torus shape space, on which we can design the whole-body coordination. Consider an $M$-dimensional motion system and its shape variable $r=$ $\left[r_{i}, r_{2}, \ldots, r_{M}\right]^{T} \in R^{M}$. Assume there are $N$ sub-systems. We term the motion of a sub-system sub-system motion. Each sub-system motion will form a closed loop gait path in the shape space $R^{M}$. The closed loop gait path in sub-system motion $i \in\{1,2, \ldots, N\}$ can be described by a function $\boldsymbol{f}_{\boldsymbol{i}}: T^{1} \rightarrow R^{M}$ such that $\boldsymbol{r}=\boldsymbol{f}_{\boldsymbol{i}}\left(\phi_{i}\right)$, where $\phi_{i} \in T^{1}$ is the gait phase variable of sub-system motion $i$. The wholebody motion can be formulated as the superposition of the sub-system motions gait paths:

$$
\boldsymbol{r}=\sum_{i=1}^{i=N} \boldsymbol{f}_{\boldsymbol{i}}\left(\phi_{i}\right)
$$

We define the sub-system motion phase variable as $\boldsymbol{\Phi}=$ $\left[\phi_{1}, \phi_{2}, \ldots, \phi_{N}\right]^{T} \in T^{N}$. We can relate the $\dot{\boldsymbol{\Phi}}$ to the shape velocity $\dot{\boldsymbol{r}}$ by the Jacobian matrix [23] $\boldsymbol{J}_{\boldsymbol{r}, \boldsymbol{\Phi}}$ :

$$
\dot{\boldsymbol{r}}=\left[\begin{array}{llll}
\frac{d f_{1}\left(\phi_{1}\right)}{\boldsymbol{d} \phi_{1}} & \frac{d f_{2}\left(\phi_{2}\right)}{\boldsymbol{d} \phi_{2}} & \ldots & \frac{d f_{N}\left(\phi_{N}\right)}{\boldsymbol{d} \phi_{N}}
\end{array}\right] \dot{\boldsymbol{\Phi}}=\boldsymbol{J}_{\boldsymbol{r}, \boldsymbol{\Phi}} \dot{\boldsymbol{\Phi}},
$$

With Eq.(6) in hand, we can rewrite Eq.(1):

$$
\begin{aligned}
\xi & =A(r) \dot{r}=A\left(\sum_{i=1}^{i=N} f_{i}\left(\phi_{i}\right)\right) \dot{r} \\
& =A\left(\sum_{i=1}^{i=N} f_{i}\left(\phi_{i}\right)\right) J_{r \Phi} \dot{\Phi}=A^{\prime}(\Phi) \dot{\Phi}
\end{aligned}
$$

where $A^{\prime}(\Phi)$ is the new local connection matrix mapping the differential of the sub-system motion phase variable, $\dot{\Phi}$, to the body velocity $\boldsymbol{\xi}$.

A properly designed gait in the sub-system motion phase variable space ( $N$-dimensional torus shape space) will then lead to coordinated whole-body motion. That is, we simplify the whole-body motion gait design problem in the original $M$ dimensional Euclidean space $R^{M}$ to the $N$-dimensional torus sub-system motion phase variable space $T^{N}$.

2) Gait design: In this section, we propose a method to design a gait path in an $N$-dimensional torus shape space. The gait path in the $N$-dimensional sub-system motion phase variable space can be described by a gait function $H: T^{1} \rightarrow$ $T^{N}$, such that: $\boldsymbol{\Phi}=\left[h_{1}\left(\phi_{0}\right), h_{2}\left(\phi_{0}\right), \ldots, h_{N}\left(\phi_{0}\right)\right]^{T} . \phi_{0} \in T^{1}$ is the global gait phase of the motion in sub-system motion phase variable space. 
We assume that all the functions $h_{i}$ are homeomorphism mapping, such that $h^{-1} \circ h$ is the identity mapping. Under this assumption, all sub-system motions will operate at the same frequency (more formally, the winding number of the gait path must be $[1,1, \ldots, 1]$ in $N$-dimensional torus space).

While there are $N$ functions in $H$, there are only $N-1$ independent functions in $H$ (dimension of $\boldsymbol{\Phi} \in T^{N}$ space minus the dimension of $\phi_{0} \in T^{1}$ space), allowing one constraint equation on $H$. For simplicity, we fix $h_{1}(x):=x$, i.e., $\phi_{1}:=\phi_{0}$. Note that our method is permutation invariant.

For simplicity, in this work we assume that all the functions in $H$ are linear mapping, such that $h_{i}(x)=x+\phi_{i}$, where $\phi_{i}$ is the phase offset of the sub-system motion $i$. In these cases, only the phase offsets between sub-system motions are subject to optimization in the whole-body coordination gait design. For more complex systems where linear mapping cannot produce the effective whole-body coordination, the mapping function prescription can be extended to sigmoidal, quadrutic, cubic or other homeomorphism mappings.

We start the optimization with a naive initial guess on the gait function $H$ that all functions in $H$ are identity mapping, i.e., $h_{i}(x)=x$. We then decompose the $N$-dimensional torus shape space into $N-1$ two-dimensional torus shape spaces $\left[\phi_{0}, \phi_{i}\right]^{T}$, where $i \in\{2,3, \ldots N\}$. Next, we optimize one independent function $h_{i} \subset H$ in the two-dimensional torus shape space composed of $\left[\phi_{0}, \phi_{i}\right]^{T} \in T^{2}$. Then, we take an approach similar to coordinate descent methods, where at each step, we optimize and update one function in $H$ and iterate among all the independent functions in $H$ until convergence. In the converged gait function $H^{*}$, every independent function $h_{i}^{*} \subset H^{*}$ should be optimized in its corresponding twodimensional torus shape space composed of $\left[\phi_{0}, \phi_{i}\right]^{T}$.

The most challenging part in our proposed gait optimization tool is to find the optimized mapping $h_{i}$ in the two-dimensional torus shape space $\left[\phi_{0}, \phi_{i}\right]^{T}$. The detailed optimization steps are presented in the remainder of this section.

To optimize each independent function $h_{i} \subset H$, we first relate the two-dimensional shape variable $\left[\phi_{0}, \phi_{i}\right]^{T}$ to the sub-system motion phase variable $\boldsymbol{\Phi}$ such that $\boldsymbol{\Phi}=$ $\left[h_{1}\left(\phi_{0}\right), h_{2}\left(\phi_{0}\right), \ldots, h_{i-1}\left(\phi_{0}\right), \phi_{i}, h_{i+1}\left(\phi_{0}\right), \ldots, h_{N}\left(\phi_{0}\right)\right]^{T}$. Next, we relate the differentials of $\phi_{0}$ and $\phi_{i}$ to the $s u b$ system motion phase variable differential by a Jacobian matrix $\boldsymbol{J}_{\boldsymbol{\Phi},\left[\phi_{0}, \phi_{i}\right]^{T}:}$

$$
\dot{\boldsymbol{\Phi}}=\boldsymbol{J}_{\boldsymbol{\Phi},\left[\phi_{0}, \phi_{i}\right]^{T}}\left[\begin{array}{l}
\dot{\phi}_{0} \\
\dot{\phi}_{i}
\end{array}\right]
$$

With Eq.(8) in hand, we can rewrite Eq.(7) as

$$
\begin{aligned}
\boldsymbol{\xi}=\boldsymbol{A}^{\prime}(\boldsymbol{\Phi}) \dot{\boldsymbol{\Phi}} & \left.=\boldsymbol{A}^{\prime}\left(\left[\begin{array}{ll}
\phi_{0} & \phi_{i}
\end{array}\right]^{T}\right) \boldsymbol{J}_{\boldsymbol{\Phi},\left[\phi_{0},\right.} \phi_{i}\right]^{T}\left[\begin{array}{c}
\dot{\phi}_{0} \\
\dot{\phi}_{i}
\end{array}\right] \\
& =\boldsymbol{A}_{\boldsymbol{H}}^{[i]}\left(\left[\begin{array}{ll}
\phi_{0} & \phi_{i}
\end{array}\right]^{T}\right)\left[\begin{array}{c}
\dot{\phi}_{0} \\
\dot{\phi}_{i}
\end{array}\right]
\end{aligned}
$$

where $\boldsymbol{A}_{\boldsymbol{H}}^{[\boldsymbol{i}]}$ is the new local connection matrix. The subscript $H$ denotes that we need the gait function $H$ to derive the local connection matrix. The superscript $[i]$ denotes that we separated $h_{i}$ from $H$ in deriving the local connection matrix.

Following the method discussed in Section II-A, we construct the height function (given by $\boldsymbol{\nabla} \times \boldsymbol{A}_{\boldsymbol{H}^{*}}^{[i]}$ ), which help us to design the mapping $h_{i}$.

3) Visualization: In this subsection, we develop the tools to visualize the contribution from a specific sub-system motion and the contribution from the coordination of two sub-system motions. We define $P_{i}$ as the set denoting the contribution from a sub-system motion $i$. The contribution from coordination between sub-system motion $i$ and $j$ can be expressed as $P_{i} \cap P_{j}$.

In Section II-B2, we construct the local connection matrix mapping the phase differentials $\dot{\phi}_{0}$ and $\dot{\phi}_{i}$ to the body velocity $\xi$. Following the approach introduced in Section II-A, we can approximate the net displacement as:

$$
\begin{aligned}
& \int_{\partial \chi} A_{H^{*}}^{[i]}\left(\left[\phi_{0} \phi_{i}\right]^{T}\right)\left[\begin{array}{c}
\dot{\phi}_{0} \\
\dot{\phi}_{i}
\end{array}\right]=\int_{\partial \chi_{1}: \phi_{0}=0} \boldsymbol{A}_{\boldsymbol{H}^{*}}^{[i]}\left(\left[\begin{array}{ll}
\phi_{\mathbf{0}} & \phi_{\boldsymbol{i}}
\end{array}\right]^{\boldsymbol{T}}\right)\left[\begin{array}{c}
\dot{\phi}_{0} \\
\dot{\phi}_{i}
\end{array}\right] \\
& +\int_{\partial \chi_{2}: \phi_{i}=0} \boldsymbol{A}_{\boldsymbol{H}^{*}}^{[i]}\left(\left[\begin{array}{ll}
\phi_{0} & \phi_{\boldsymbol{i}}
\end{array}\right]^{T}\right)\left[\begin{array}{c}
\dot{\phi}_{0} \\
\dot{\phi}_{i}
\end{array}\right] \\
& +\iint_{\chi-\chi_{1}-\chi_{2}} \nabla \times A_{H^{*}}^{[i]}\left(\left[\phi_{0} \phi_{i}\right]^{T}\right) d \phi_{0} d \phi_{i}
\end{aligned}
$$

The physical meaning of each term (right hand side) in Eq.(10) can be interpreted in a similar manner to our analysis in Section II-A. The physical meaning of the first term (right hand side) in Eq.(10) is the displacement solely from the motion in sub-system $i$, while keeping $\phi_{0}$ constant at initial position. Note that all other sub-system motions have been coupled to $\phi_{0}$ by $H^{*}$ in our derivation of local connection matrix $\boldsymbol{A}_{\boldsymbol{H}^{*}}^{[\boldsymbol{i}]}$. Keeping $\phi_{0}$ constant at initial position is equivalent to keeping all other sub-system motions stopped at initial position. Thus, the first term (right hand side) in Eq.(10) can be interpreted as the displacement solely from sub-system motion $i$, while keeping all other motion systems stopped at initial position, i.e., $P_{i} \cap P_{[i]}^{C}$, where $P_{[i]}=P_{1} \cup P_{2} \cup \ldots \cup P_{i-1} \cup P_{i+1} \cup \ldots \cup P_{N}$. The subscript [i] in $P_{[i]}$ denotes that $P_{[i]}$ is the union of the contribution of all other sub-system motions except sub-system motion $i$. Since only one sub-system motion is involved in the first term (right hand side) in Eq.(10), it is then a precomputed constant, independent from the sub-system motion coordination.

The physical meaning of the third term (right hand side) in Eq.(10) is the additional displacement from coordination between sub-system motion $i$ and all other sub-system motions, i.e., $P_{i} \cap P_{[i]}$.

Since $P_{i}=\left(P_{i} \cap P_{[i]}^{C}\right) \cup\left(P_{i} \cap P_{[i]}\right), P_{i}$ can be visually analyzed by the surface integral over the height function $\nabla \times A_{H^{*}}^{[i]}$ plus a pre-computed constant.

Next, we develop the tool to visualize the contribution from coordination of two specific sub-system motions $i$ and $j$, i.e., $C_{i} \cap C_{j}$. In order to construct $C_{i} \cap C_{j}$, we first assign $h_{j}=0$ such that in this scenario, sub-system motion $j$ is stopped. Following our approach in section II-B2, we construct a two- 
dimensional shape variable $\left[\phi_{0}, \phi_{i}\right]$ and relate it to the subsystem motion phase variable $\boldsymbol{\Phi}^{[j]}$ (the superscript $[j]$ denotes that $\phi_{j}$ is not involved in $\boldsymbol{\Phi}^{[j]}$ ) as:

$$
\begin{array}{r}
\Phi^{[j]}=\left[h_{1}^{*}\left(\phi_{0}\right), \ldots, h_{i-1}^{*}\left(\phi_{0}\right), \phi_{i}, h_{i+1}^{*}\left(\phi_{0}\right), \ldots,\right. \\
\left.\ldots, h_{j-1}^{*}\left(\phi_{0}\right), 0, h_{j+1}^{*}\left(\phi_{0}\right), h_{N}^{*}\left(\phi_{0}\right)\right]^{T}
\end{array}
$$

Given Eq.(11), we can relate the differential of $\phi_{0}$ and $\phi_{i}$ to the sub-system motion phase variable differential $\Phi^{[j]}$ by a Jacobian matrix $\boldsymbol{J}_{\boldsymbol{\Phi}^{[j]},\left[\phi_{0}, \phi_{i}\right]^{T}}$ :

$$
\dot{\boldsymbol{\Phi}}^{[j]}=\boldsymbol{J}_{\boldsymbol{\Phi}^{[j]},\left[\phi_{0}, \phi_{i}\right]^{T}}\left[\begin{array}{c}
\dot{\phi}_{0} \\
\dot{\phi}_{i}
\end{array}\right]
$$

Note that motion associated with the sub-system motion phase variable $\boldsymbol{\Phi}^{[j]}$ in Eq.(12) does not include the motion in sub-system $j$. Similar to Eq.(7), we can then relate it to the shape velocity:

$$
\begin{aligned}
& \boldsymbol{\xi}^{[j]}=\boldsymbol{A}\left(\sum_{k=1}^{k=j-1} \boldsymbol{f}_{k}\left(\phi_{k}\right)+\sum_{k=j+1}^{k=N} \boldsymbol{f}_{k}\left(\phi_{k}\right)\right) \dot{\boldsymbol{r}} \\
& \left.=\boldsymbol{A}^{\prime}\left(\boldsymbol{\Phi}^{[j]}\right) \dot{\boldsymbol{\Phi}}^{[j]}=\boldsymbol{A}^{\prime}\left(\left[\phi_{0} \phi_{i}\right]^{T}\right) \boldsymbol{J}_{\boldsymbol{\Phi}^{[j]},\left[\phi_{0}, \phi_{i}\right.}\right]^{T}\left[\begin{array}{c}
\dot{\phi}_{0} \\
\dot{\phi}_{i}
\end{array}\right] \\
& =\boldsymbol{A}_{H^{*}}^{[i][j]}\left(\left[\begin{array}{ll}
\phi_{0} & \phi_{i}
\end{array}\right]^{T}\right)\left[\begin{array}{c}
\dot{\phi}_{0} \\
\dot{\phi}_{i}
\end{array}\right]
\end{aligned}
$$

where $\boldsymbol{\xi}^{[j]}$ denotes the body velocity (the superscript $[j]$ in $\boldsymbol{\xi}^{[j]}$ denotes that sub-system motion $j$ is not coupled in the body velocity) and $\boldsymbol{A}_{\boldsymbol{H}^{*}}^{[i][j]}$ denotes the new local connection. The superscript $[i][j]$ in $A_{H^{*}}^{[i][j]}$ denotes that we separated $h_{i}$ and $h_{j}$ from $H^{*}$ in deriving $A_{H^{*}}^{[i][j]}$. With Eq.(13), we can rewrite Eq.(3) as:

$$
\begin{aligned}
& \int_{\partial \chi} A_{H^{*}}^{[i][j]}\left(\left[\phi_{0} \phi_{i}\right]^{T}\right)\left[\begin{array}{c}
\dot{\phi}_{0} \\
\dot{\phi}_{i}
\end{array}\right]=\int_{\partial \chi_{1}: \phi_{0}=0} A_{H^{*}}^{[i][j]}\left(\left[\begin{array}{ll}
\phi_{0} & \phi_{i}
\end{array}\right]^{T}\right)\left[\begin{array}{c}
\dot{\phi}_{0} \\
\dot{\phi}_{i}
\end{array}\right] \\
& +\int_{\partial \chi_{2}: \phi_{i}=0} \boldsymbol{A}_{H^{*}}^{[i][j]}\left(\left[\begin{array}{ll}
\phi_{0} & \phi_{i}
\end{array}\right]^{T}\right)\left[\begin{array}{c}
\dot{\phi}_{0} \\
\dot{\phi}_{i}
\end{array}\right] \\
& +\iint_{\chi-\chi_{1}-\chi_{2}} \nabla \times \boldsymbol{A}_{H^{*}}^{[i][j]}\left(\left[\begin{array}{ll}
\phi_{0} & \phi_{i}
\end{array}\right]^{T}\right) \boldsymbol{d} \phi_{0} d \phi_{i}
\end{aligned}
$$

Similar to our analysis in Section II-B3, we can interpret the physical meaning of the third term (right hand side) in Eq.(14) as the additional displacement resulting from the coordination between sub-system motion $i$ and all other sub-system motions coupled to $\phi_{0}$ in Eq.(11), where we assume that sub-system motion $j$ is stopped. In this way, we can express the physical meaning of the third term (right hand side) in Eq.(14) as $P_{i} \cap$ $\left(P_{[i]}-P_{j}\right)$.

Given Eq.(10) and Eq.(14), we can construct $P_{i} \cap P_{j}$. We subtract the third term (right hand side) in Eq.(14) from the third term (right hand side) in Eq.(10), giving us:

$$
\begin{aligned}
& P_{i} \cap P_{j}=P_{i} \cap P_{[i]}-P_{i} \cap\left(P_{[i]}-P_{j}\right) \\
& =\iint_{\chi_{-\chi_{1}-\chi_{2}}} \nabla \times\left(\boldsymbol{A}_{\boldsymbol{H}^{*}}^{[i]}\left(\left[\begin{array}{c}
\dot{\phi}_{\mathbf{0}} \\
\dot{\phi}_{\boldsymbol{i}}
\end{array}\right]\right)-\boldsymbol{A}_{\boldsymbol{H}^{*}}^{[i][j]}\left(\left[\begin{array}{c}
\dot{\phi}_{\mathbf{0}} \\
\dot{\phi}_{\boldsymbol{i}}
\end{array}\right]\right)\right) \boldsymbol{d} \phi_{0} \boldsymbol{d} \phi_{i}
\end{aligned}
$$
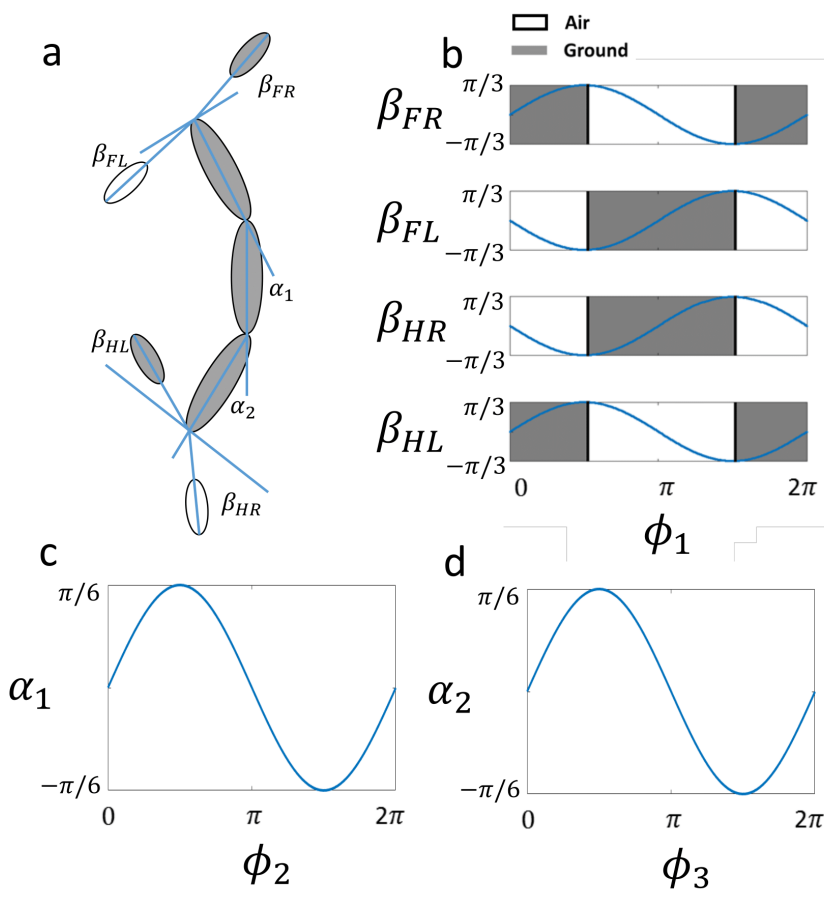

Fig. 3. The model for our robot and its sub-system motions. (a) The shape variables of a robot with a long articulated body and four legs. The body undulation joint angle, $\alpha_{i}$, and leg shoulder joint angle $\beta_{i}$ are labelled. Segments in solid ellipses are in contact with the ground; segments in open ellipses are in the air. (b) Leg sub-system motion. The leg sub-system motion is prescribed by a periodic variable $\phi_{1}$. The row of eight boxes indicate the contact state of the leg during the phase of the gait, where filled gray means ground contact and open white means in the air. The blue curves indicate the leg shoulder joint angles. (c) Upper back sub-system motion. The upper back sub-system motion is prescribed by a periodic variable $\phi_{2}$. (d) Lower back sub-system motion. The lower back sub-system motion is prescribed by a periodic variable $\phi_{3}$

In this way, we can visualize $P_{i} \cap P_{j}$ as the surface integral over the constructed height function $\nabla \times\left(A_{H^{*}}^{[i]}-A_{H^{*}}^{[i][j]}\right)$.

\section{EXAMPLE: GAIT DESIGN FOR A ROBOT WITH A LONG ACTUATED BODY AND INTERMEDIATE-SIZED LEGS}

In this section, we apply our proposed hierarchical framework to design gaits for a robot with a long actuated body and intermediate-sized legs. Existing approaches to design gaits for quadrupedal systems can be found in [27]. We validate our proposed hierarchical framework by robot experiments using a robophysical approach $[1,19]$. We first test the whole-body motion resulting from the two common approaches to design quadrupedal body undulation. We next apply our proposed framework to design whole-body motion and visualize the kinematics of whole-body coordination. Finally, we verify our theoretical predictions by robot experiments and RFT simulation.

\section{A. Experimental set-up}

We built an open-loop, servo-driven, 3D-printed robot (450 $\mathrm{g}, \sim 40 \mathrm{~cm}$ long) with a long actuated body and intermediatesized legs (Fig.1), and tested its performance on granular media ( $\sim 1 \mathrm{~mm}$ diameter poppy seeds). Each leg (front 


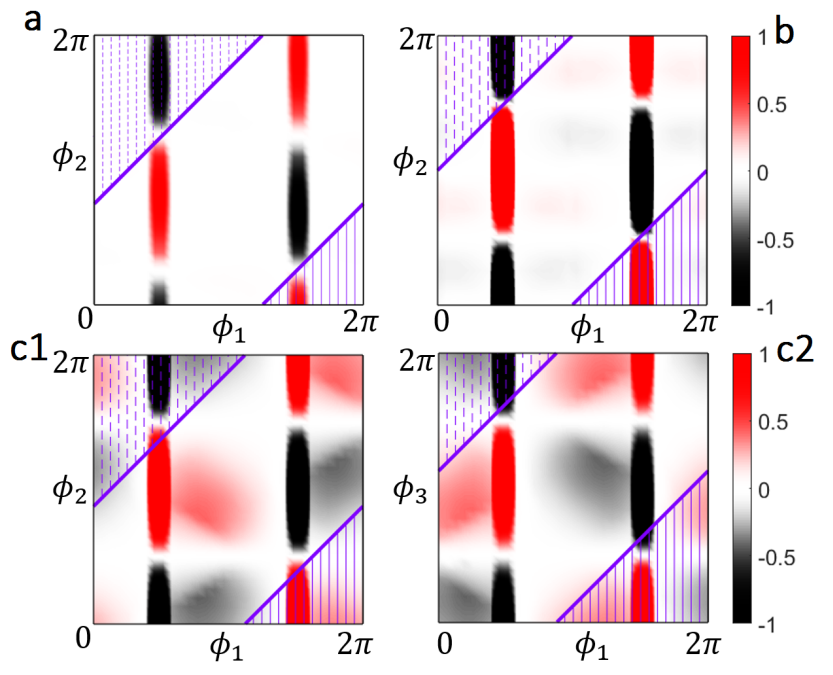

Fig. 4. Height functions (a) The height function presenting the coordination of snake-like body undulation and leg movement. (b) The height function presenting the coordination of lizard-like body undulation and leg movement. (c1) The height function presenting the coordination of upper back and leg movement. (c2) The height function presenting the coordination of lower back and leg movement. In all illustrations, the solid blue curve represents the optimized gait path.

right $F R$, front left $F L$, hind right $H R$ and hind left $H L$ ) has two DoFs, with one controlling the planar shoulder joint angle $\beta_{i}$ and the other controlling the binary ground contact $\delta_{i}$. The body consists of three segments, connected by two independently-actuated joints (upper back joint angle $\alpha_{1}$ and lower back joint angle $\alpha_{2}$ ), and maintains continuous contact with the ground (Fig. 3).

The robot executed a programmed set of movements (following the gait input) to walk on the loosely-packed poppy seeds. Throughout the experiment, four Optitrack Flex13 cameras recorded the positions of the infrared-reflective markers on the robot. At the end of each experiment, the robot's final position was identified. Before each experiment, an airfluidized bed erased the footprints and allowed the seeds to be reset into a loosely-packed state.

\section{B. Sub-system motions}

We decompose the motion of the robot into three subsystem motions: the leg sub-system motion, the upper back sub-system motion and the lower back sub-system motion.

1) Leg sub-system motion: We assume that the leg subsystem motion follows a trot gait pattern, a typical type of leg movements given from prior work [9]. The trot is a two-beat gait with diagonally paired leg movements. The fore right leg is always in phase with the hind left leg while the fore left leg is always in phase with the hind right leg. In Fig. 3b, we show that the shoulder joint angles and the leg contact patterns of all legs are prescribed by a periodic variable $\phi_{1}$.

2) Upper back sub-system motion: We prescribe the upper back joint angle, $\alpha_{1}$, as a sinusoidal function of the gait phase $\phi_{2}$, as illustrated in Fig. 3c. While the upper back motion system itself cannot generate effective locomotion, it
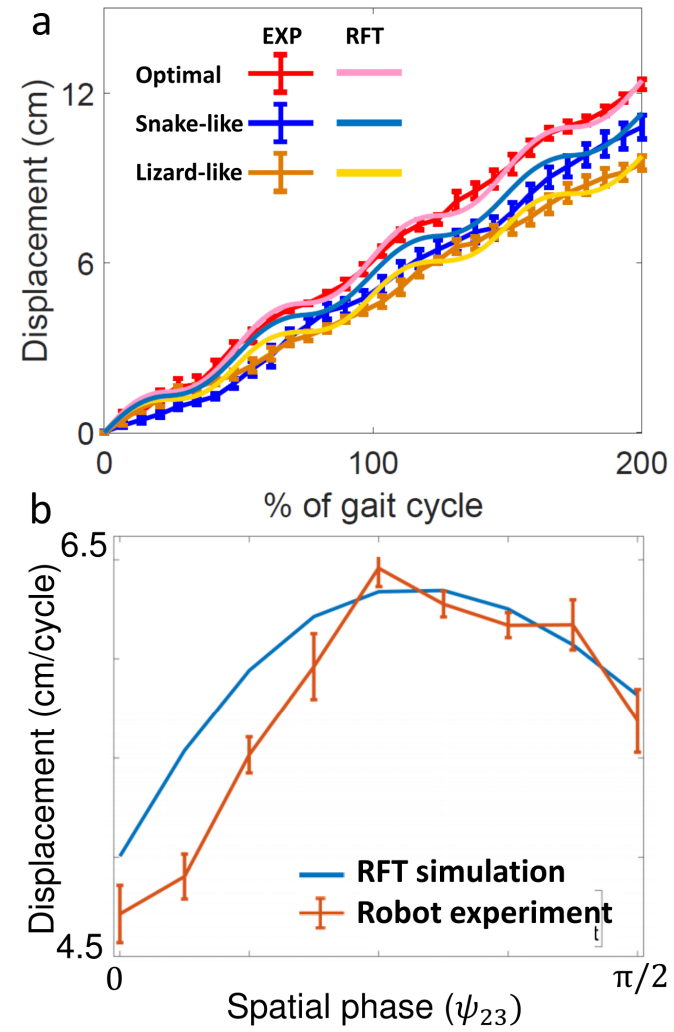

Fig. 5. Robot experiment and RFT simulation results (a) The primary data of displacement from robot experiments (solid lines with error bars) and RFT simulation (solid lines) plotted as a function of gait percentage over two gait cycles for snake-like body undulation (blue), optimal body undulation (red) and lizard-like body undulation (yellow). (b) The displacement is plotted as a function of spatial phase. The red curve with error bars shows the robot experiment results. The blue curve shows the RFT simulation results.

can improve the locomotion performance by coordinating with the leg sub-system motion and the lower back sub-system motion.

3) Lower back sub-system motion: Similar to upper back sub-system motion, we prescribe the lower back joint angle, $\alpha_{2}$, as a sinusoidal function of the gait phase $\phi_{3}$, as illustrated in Fig. 3d. While the lower back motion system itself cannot generate effective locomotion, it can improve the locomotion performance by coordinating with the leg sub-system motion and the upper back sub-system motion.

\section{Whole body motion gait design}

Body undulation plays an important role in whole-body motion. In some cases where the leg drag is dominating (lizard-like locomotion), the body undulation will form a standing wave to improve the self-propulsion by leg-ground interaction [11, 27]. In other cases where the body drag is dominating (snake-like locomotion), the body undulation will form a traveling serpenoid wave to generate self-propulsion by body-ground interaction [8]. In this section, we explore the role of body undulation for a quadrupedal robot with a long actuated body and intermediate-sized legs.

We assume all the mapping functions are linear, i.e., $\phi_{i}=$ $\phi_{j}+\psi_{i j}$, where $\psi_{i j}$ is the phase offset between the sub-system 
motion $i$ and $j$. We define the spatial phase as the phase shift between the upper back and lower back, i.e., $\psi_{23}$. The spatial phase characterizes the wave pattern of the body undulation.

In our robot experiments, we found that the optimal spatial phase, intermediate between lizard-like and snake-like, maximizes the displacement. The optimized whole-body motion will leverage the self-propulsion of leg-ground contact and the self-propulsion of body-ground contact.

1) Snake-like body undulation: The long actuated body of the robot can generate self-propulsion from body-ground interaction. Hatton et al. [8] studied the motion of a threelink swimmer on granular media, where the body undulation follows the traveling serpenoid wave. We call the traveling serpenoid wave body undulation snake-like body undulation. In snake-like body undulation, we couple the upper and lower back sub-system motions such that they form a traveling serpenoid wave propagated from head to tail. Specifically, the phase of lower back sub-system motion is $\pi / 2$ ahead of the phase of upper back sub-system motion, i.e., the spatial phase of snake-like body undulation is $\psi_{23}^{\text {snake }}=\pi / 2$.

Next, we seek to coordinate the traveling serpenoid wave body undulation with the leg movement, which is characterized by $\psi_{12}^{\text {snake }}$. Following the steps in section II-B2, we can calculate the height function on the shape space composed of $\left[\phi_{1}, \phi_{2}\right]^{T}$ and then design the proper whole-body coordination gait. The height function and the optimized whole-body coordination gait for snake-like body undulation is shown in Fig 4a. To summarize, in snake-like body undulation, we have $\psi_{12}^{\text {snake }}=0.75 \pi, \psi_{13}^{\text {snake }}=1.25 \pi$ and $\psi_{23}^{\text {snake }}=0.5 \pi$.

2) Lizard-like body undulation: The long actuated body can, on the other hand, help to improve the self-propulsion by leg-ground interaction. Zhong et al. [27] studied the motion of a quadrupedal robot with a single DoF in the back. Ijspeert et al. [11] showed that standing wave body undulation can be coordinated with leg movements during walking. We call the standing wave body undulation lizard-like body undulation. Although the lizard-like body undulation itself does not lead to effective motion, it can be coordinated with leg movement to improve locomotion performance.

In lizard-like body undulation, we couple the upper and lower back such that they form a standing wave. Specifically, lower back sub-system motion is always in phase with upper back sub-system motion, i.e., $\phi_{2}=\phi_{3}$, such that spatial phase $\psi_{23}^{\text {lizard }}=0$.

Next, we seek to coordinate the standing wave body undulation with the leg movement, which is characterized by $\psi_{12}^{\text {lizard }}$. Following the steps in section II-B2, we can calculate the height function on the two-dimensional shape space composed of $\left[\phi_{1}, \phi_{2}\right]^{T}$ and then design the proper wholebody coordination gait. The height function and the optimized whole-body coordination gait for lizard-like body undulation is shown in Fig 4b. To summarize, in lizard-like body undulation, we have $\psi_{12}^{\text {lizard }}=\pi, \psi_{13}^{\text {lizard }}=\pi$ and $\psi_{23}^{\text {lizard }}=0$.

3) Optimal body undulation: In this subsection, we consider the upper back and lower back as two independent subsystem motions. With the methods described in section II-B2, we can design the whole-body motion gait in the threedimensional sub-system motion phase space. Fig. $4 \mathrm{c}$ shows the converged optimal gaits on their corresponding height functions. In optimal body undulation, we have $\psi_{12}^{\text {optimal }}=1.13 \pi$, $\psi_{13}^{\text {optimal }}=0.88 \pi$ and $\psi_{23}^{\text {optimal }}=0.25 \pi$. We observe that spatial phase in optimal undulation $\psi_{23}^{\text {optimal }}$ is intermediate between $\psi_{23}^{\text {snake }}$ and $\psi_{23}^{\text {lizard }}$.

The theory-predicted optimal body undulation for our robot is neither traveling serpenoid wave nor standing wave. Instead, the intermediate spatial space in the spectrum, from lizardlike to snake-like, will maximize the forward displacement. To verify our theory, we sampled the spectrum and tested the the resulting whole-body motion on the robot experiments and RFT simulation. Both robot experiments and RFT simulation agree well with our theory prediction.

\section{Visualization}

In order to visualize the contribution from each individual sub-system motion, we present a Venn diagram for sub-system motions in Table I. There are three major sets: $P_{l e g}$, contribution from the leg movement (blue circle), $P_{u p}$, contribution from the upper back (red circle), and $P_{\text {low }}$, the contribution from the lower back (yellow circle). The intersection of three sets creates 4 additional regions (for a total of 7). The physical meaning of each region is listed in Table I.

Note that regions 1, 3 and 6 are the contribution solely from upper back, lower back and leg sub-system motion respectively. The contribution solely from an individual sub-system motion is a pre-computed constant, independent from any gait we prescribe. Other regions, presenting the contribution from coordination, are determined by the gait paths (characterize by the phase offset $\psi_{12}, \psi_{13}$ and $\psi_{23}$ ) we choose. In Table I, we listed the dependence of each region on the phase offsets.

Following the steps in Section II-B3, we can visualize the contribution from each region in Fig. 6. Note that in a motion system with three sub-system motions, all the regions in the Venn diagram can be visualized from the basic set operations. From Fig. 6c and Fig. 6d, we observe that optimized body undulation indeed leads to additional displacement by increasing the self-propulsion from the leg-ground interaction, similar to the role of lizard body undulation. On the other hand, Fig. 6a suggests that the slight phase offset between upper back and lower back, $\psi_{23}$, can lead to additional displacement by selfpropulsion from body-leg interaction, similar to the role of snake body undulation.

\section{E. Results}

In our robot experiments, we recorded the displacement of the robot as it implemented the different gaits. In Fig. 5a, we plotted the displacement as a function of gait percentage for the snake-like, optimal body and lizard-like body undulation gaits. In Fig. 5b, we plotted the displacement as a function of spatial phase. Both simulation and robot experiments suggest that our theoretically calculated optimal spatial phase will lead to the largest displacement. 


\begin{tabular}{|c|c|c|c|c|}
\hline Venn Diagram & Region & Definition & Physical meaning & Determined by \\
\hline \multirow{7}{*}{$\begin{array}{l}\text { Upper back Lower back } \\
\text { (UB) }\end{array}$} & 1 & $P_{u p} \cap\left(P_{\text {leg }} \cup P_{\text {low }}\right)^{C}$ & solely from UB & - \\
\hline & 2 & $\left(P_{\text {up }} \cap P_{\text {low }}\right) \cap P_{\text {leg }}^{C}$ & from coordination of UB and LB & $\psi_{23}$ \\
\hline & 3 & $P_{\text {low }} \cap\left(P_{\text {leg }} \cup P_{u p}\right)^{C}$ & solely from LB & - \\
\hline & 4 & $P_{\text {low }} \cap P_{\text {leg }} \cap P_{u p}$ & whole body coordination & $\psi_{12}$ and $\psi_{13}$ \\
\hline & 5 & $\left(P_{\text {leg }} \cap P_{\text {low }}\right) \cap P_{u p}^{C}$ & from coordination of LB and legs & $\psi_{13}$ \\
\hline & 6 & $P_{\text {leg }} \cap\left(P_{\text {up }} \cup P_{\text {low }}\right)^{C}$ & solely from legs & - \\
\hline & 7 & $\left(P_{l e g} \cap P_{l o w}\right) \cap P_{u p}^{C}$ & from coordination of UB and legs & $\psi_{12}$ \\
\hline
\end{tabular}

TABLE I

DEFINITION AND PHYSICAL MEANING OF EACH OF THE REgIONS IN THE VENN DIAGRAM FOR OUR ROBOT.

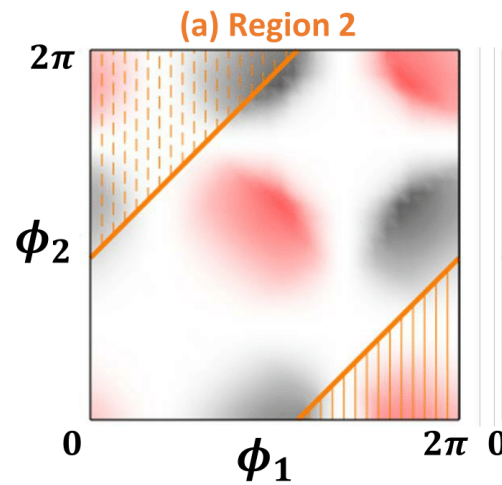

(b) Region 4

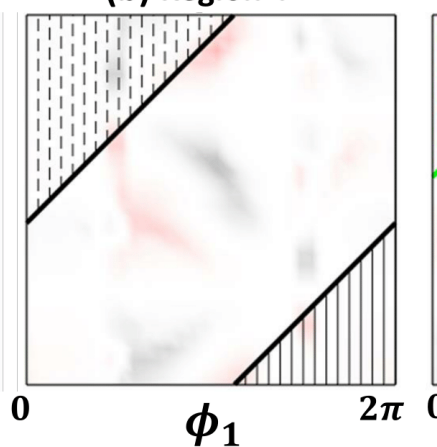

(c) Region 5

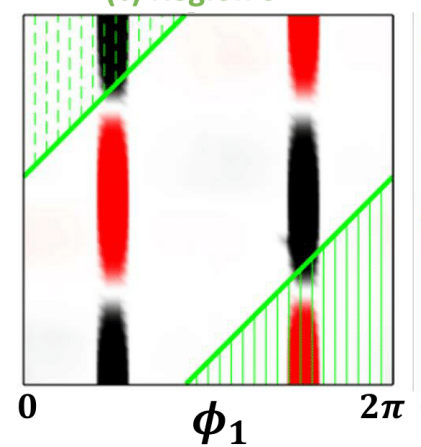

(d) Region 7

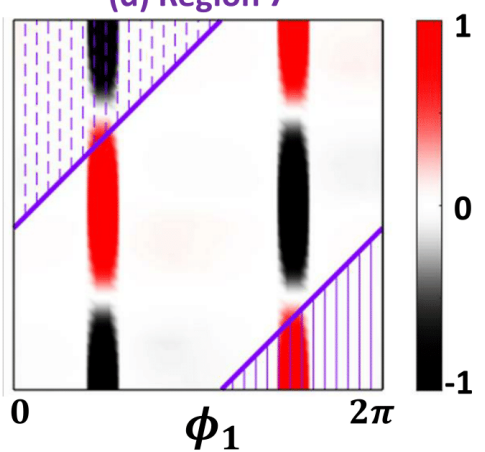

Fig. 6. Visualization of the contribution from individual motion sub system and the contribution from the coordination. Each region in the Venn diagram can be visualized by the surface integral over the corresponding height function.

We observe that the optimal body undulation spatial phase decreases from $\pi / 2$ to 0 for a robot with a long actuated body and infinitesimal-sized legs (like a snake), a robot with a long actuated body and intermediate-sized legs, and a robot with a long actuated body and long legs (like a lizard). Such spatial phase decreases could be associated with the changes in leg sizes. We hypothesize that the evolution of leg size (limblessness of snake) is associated with the changes in their corresponding locomotion patterns. This framework may provide insight into the hypothesis on the correspondence between form and function [18].

\section{CONCLUSION}

In this paper, we presented a new framework for designing locomotion by coordinating motions of different subsystems, as well as visualizing the kinematics of the resulting whole-body motion. Our framework allows us to design, optimize, and visualize the whole-body motion. We applied our framework to design a whole-body motion for quadrupedal robots. A quadrupedal robot with a long actuated body and infinitesimally small legs (snake-like) mainly utilizes its bodyground interaction to generate self-propulsion. In contrast, the quadrupedal robot with a long actuated body and long legs (lizard-like) mainly unitizes its leg-ground interaction to generate self-propulsion. Our quadrupedal robot, with a long actuated body and intermediate-sized legs, combines both approaches to coordinate the self-propulsion from both body-leg interaction and leg-ground interactions to generate efficient locomotion. Our theory-predicted optimized wholebody motion agrees well with the presented robot experiments and RFT simulation.

In future work, we will further explore the underlying link between our hierarchical gait design framework and the way animals have optimized their locomotion through evolution. Specifically, we believe there could be interesting similarities between our framework and the emergence of locomotion patterns in certain quadrupeds (e.g., lizards, skinks), as well as centipedes and millipedes. In doing so, we will study how biological systems coordinate their $\mathrm{HDoF}$ motion systems and adapt to different environments and leg/body morphologies.

Although this paper only considered cases where the hierarchy had a depth of one (i.e., no sub-sub-systems), we believe that our approach can be used recursively to optimize locomotion for a robot decomposed into a deeper hierarchy of sub-systems, sub-sub-systems, etc. Additionally, this framework can also be applied to complex high-dimensional motion systems where many sub-systems exist. For the future, we will extend our work to study real-time motion planning in response to possible sub-system failures. That is, when some joints fail to be actuated, we envision that our framework will be able to design a new whole-body motion in response to these new constraints. Additionally, we will investigate how our framework can be used to analyze animal locomotion with numerous sub-systems in complex environments. 


\section{REFERENCES}

[1] Jeffrey Aguilar, Tingnan Zhang, Feifei Qian, Mark Kingsbury, Benjamin McInroe, Nicole Mazouchova, Chen Li, Ryan Maladen, Chaohui Gong, Matt Travers, et al. A review on locomotion robophysics: the study of movement at the intersection of robotics, soft matter and dynamical systems. Reports on Progress in Physics, 79 (11):110001, 2016.

[2] Gordon J Berman, Daniel M Choi, William Bialek, and Joshua W Shaevitz. Mapping the stereotyped behaviour of freely moving fruit flies. Journal of The Royal Society Interface, 11(99):20140672, 2014.

[3] Gregory S Chirikjian and Joel W Burdick. The kinematics of hyper-redundant robot locomotion. IEEE transactions on robotics and automation, 11(6):781-793, 1995.

[4] Jin Dai, Hossein Faraji, Chaohui Gong, Ross L Hatton, Daniel I Goldman, and Howie Choset. Geometric Swimming on a Granular Surface. In Robotics: Science and Systems, 2016.

[5] Chaohui Gong, Daniel I Goldman, and Howie Choset. Simplifying Gait Design via Shape Basis Optimization. In Robotics: Science and Systems, 2016.

[6] Chaohui Gong, Matthew J Travers, Henry C Astley, $\mathrm{Lu} \mathrm{Li}$, Joseph R Mendelson, Daniel I Goldman, and Howie Choset. Kinematic gait synthesis for snake robots. The International Journal of Robotics Research, 35(1-3): 100-113, 2016.

[7] Chaohui Gong, Julian Whitman, Jaskaran Grover, Baxi Chong, Richard Ren, and Howie Choset. Geometric motion planning for systems with toroidal and cylindrical shape spaces. In Dynamic Systems and Control Conference, 2018.

[8] Ross L Hatton, Yang Ding, Howie Choset, and Daniel I Goldman. Geometric visualization of self-propulsion in a complex medium. Physical review letters, 110(7): 078101, 2013.

[9] Milton Hildebrand. Symmetrical gaits of horses. science, 150(3697):701-708, 1965.

[10] Shigeo Hirose. Biologically inspired robots: snake-like locomotors and manipulators, volume 1093. Oxford University Press Oxford, 1993.

[11] Auke Jan Ijspeert, Alessandro Crespi, Dimitri Ryczko, and Jean-Marie Cabelguen. From swimming to walking with a salamander robot driven by a spinal cord model. science, 315(5817):1416-1420, 2007.

[12] Shoshichi Kobayashi and Katsumi Nomizu. Foundations of differential geometry, volume 1. Interscience publishers New York, 1963.

[13] Chen Li, Tingnan Zhang, and Daniel I Goldman. A terradynamics of legged locomotion on granular media. Science, 339(6126):1408-1412, 2013.

[14] Jerrold E Marsden and Tudor S Ratiu. Introduction to mechanics and symmetry: a basic exposition of classical mechanical systems, volume 17. Springer Science \&
Business Media, 2013.

[15] Hamidreza Marvi, Chaohui Gong, Nick Gravish, Henry Astley, Matthew Travers, Ross L Hatton, Joseph R Mendelson, Howie Choset, David L Hu, and Daniel I Goldman. Sidewinding with minimal slip: Snake and robot ascent of sandy slopes. Science, 346(6206):224229, 2014.

[16] Kamilo Melo and Laura Paez. Experimental determination of control parameter intervals for repeatable gaits in modular snake robots. In Safety, Security, and Rescue Robotics (SSRR), 2014 IEEE International Symposium on, pages 1-7. IEEE, 2014.

[17] Hiromi Mochiyama, Etsujiro Shimemura, and Hisato Kobayashi. Shape control of manipulators with hyper degrees of freedom. The International Journal of Robotics Research, 18(6):584-600, 1999.

[18] John A Nyakatura, Kamilo Melo, Tomislav Horvat, Kostas Karakasiliotis, Vivian R Allen, Amir Andikfar, Emanuel Andrada, Patrick Arnold, Jonas Lauströer, John R Hutchinson, et al. Reverse-engineering the locomotion of a stem amniote. Nature, 565(7739):351, 2019.

[19] Yasemin Ozkan-Aydin, Jennifer M. Rieser, Christian M. Hubicki, William Savoie, and Daniel I. Goldman. Physics approaches to natural locomotion: Every robot is an experiment. In Shawn M. Walsh and Michael S. Strano, editors, Robotic Systems and Autonomous Platforms, Woodhead Publishing in Materials, pages 109 - 127. Woodhead Publishing, 2019.

[20] Suresh Ramasamy and Ross L Hatton. Geometric gait optimization beyond two dimensions. In American Control Conference (ACC), 2017, pages 642-648. IEEE, 2017.

[21] Shai Revzen and Daniel E Koditschek. Why we need more Degrees of Freedom. Procedia IUTAM, 20:89-93, 2017.

[22] Sarah S Sharpe, Stephan A Koehler, Robyn M Kuckuk, Miguel Serrano, Patricio A Vela, Joseph Mendelson, and Daniel I Goldman. Locomotor benefits of being a slender and slick sand swimmer. Journal of Experimental Biology, 218(3):440-450, 2015.

[23] Angus Ellis Taylor and William Robert Mann. Advanced calculus. Wiley, 1972.

[24] Matthew Tesch, Kevin Lipkin, Isaac Brown, Ross Hatton, Aaron Peck, Justine Rembisz, and Howie Choset. Parameterized and scripted gaits for modular snake robots. Advanced Robotics, 23(9):1131-1158, 2009.

[25] Matthew J Travers, Julian Whitman, Perrin Schiebel, Daniel I Goldman, and Howie Choset. Shape-Based Compliance in Locomotion. In Robotics: Science and Systems, 2016.

[26] Tingnan Zhang and Daniel I Goldman. The effectiveness of resistive force theory in granular locomotion a. Physics of Fluids, 26(10):101308, 2014.

[27] Baxi Zhong, Yasemin Ozkan Aydin, Chaohui Gong, Guillaume Sartoretti, Yunjin Wu, Jennifer Rieser, Haosen 
Xing, Jeffery Rankin, Krijn Michel, Alfredo Nicieza, et al. Coordination of back bending and leg movements for quadrupedal locomotion. In Robotics: Science and Systems, 2018. 